\title{
Performance Analysis of DTN Using Level Signal Priority Epidemic Routing Protocol
}

\author{
Ahmad Zainudin, Amang Sudarsono, Kevin Prima Pambudi \\ Department of Electrical Engineering \\ Electronic Engineering Polytechnic Institute of Surabaya \\ Kampus PENS, Jalan Raya ITS, Sukolilo 60111, Indonesia \\ E-mail: \{zai,amang\}@pens.ac.id, sir.kevinprima@gmail.com
}

\begin{abstract}
A DTN architecture consists of several nodes that are connected with high dynamic topology. The routing protocol is an important part which determine the DTN performance system. Although DTN is addressed to be tolerant of delay, a routing protocol with better performance will maximizing packet delivery rate and minimizing the delivery latency. This paper evaluate a level signal priority epidemic routing protocol for delay tolerant network architecture. Our system adopts DTN2 framework using classic epidemic and priority epidemic dynamic routing protocols. The performance of both dynamic routing is observed and compared based on throughput and delay of transmitted data. The measurement results show that the classic epidemic use more bandwith due to sending the same messages many times. The delay transmission using a level signal priority epidemic routing is smaller than classic epidemic routing protocol in all hops of the test-bed. Epidemic based on signal level routing could make traffic of network more efficient than classic Epidemic routing because of filtering system in node before sending bundle to neighbor node.
\end{abstract}

Keywords: DTN, dynamic routing, level signal priority

\section{INTRODUCTION}

The channel of wireless communication system may change dynamically and randomly. When the communication use TCP/IP protocol and the channel is down or unavailable, most likely to occur retransmission and high probability the packet data is loss for long delay transmission. TCP/IP model use end-to-end principle, which path between source and destibation is exist with continuous connection. Delay Tolerant Network (DTN) protocol can overcome the problems with long duration packet data delivery using a store and forward principle [1]. The packet data is transmitted from source to destination through some carrier mobile nodes. When the link from source to carrier node available, the packet data is 
delivered to carrier node. But when the channel between another carrier node is down or unavailable the packet data is stored in the last carrier node. The packet data is forwarded if the link to destination available.

DTN protocol is addressed for network characteristic with intermittent connectivity, long and variable delay, high error rates and no guarantee of end-to-end connectivity between source and destination [2]. The DTN architecture use store and forward message switching with implements a bundle layer between transport layer and application layer. A DTN architecture consists of several nodes that are connected with high dynamic topology. The routing protocol is an important part which determine the DTN performance system. Although DTN is addressed to be tolerant of delay, a routing protocol with better performance will maximizing packet delivery rate and minimizing the delivery latency [3].

The DTN routing protocols based on property is used are classified into flooding families and forwarding families. The epidemic routing protocol including in flooding families. In this routing protocol, each node copy the packet and sent to the another node to achieve the destination. Meanwhile, the forwarding families use knowledge about network information to find the destination node. The forwarding routing is more efficient than the flooding based routing to make routing decision [4]. Beside that forwarding routing has better performance with minimum resource space and network bandwidth than flooding routing.

In this paper, the performance of classic epidemic and priority epidemic routing protocol for delay tolerant network architecture are campared. The system is implemented use DTN2 framework. The performance system is observed based on throughput and delay of transmitted data. The measurement results show that the average of throughput and delay transmission using a level signal priority epidemic routing is smaller than classic epidemic routing protocol in all hops of the test-bed.

\section{RELATED WORKS}

Rango et. al. [4] compare the n-epidemic routing and a novel strategies of epidemic routing protocol called Energy Aware Epidemic Routing (EAER). The n-epidemic routing is a optimization of sending messages from the node to its neighbor which a node can start to transmit only when it has at least $n$ neighbors. The EAER routing protocol is enhancement of n-epidemic routing based on energy consumption. This strategies use the node density estimation and the nodes energy levels. The advantages of these enhancement protocols to reduce energy consumption and increase message delivery probability. The parameters that compared including delivery probability, average hop count, data delivery delay and energy consumption. The comparation of these routing algorithm show that when mobile nodes have good energy budget, more data delivery can be allowed and the transmission probability can be increased. 
Bajpai et. al. [5] propose a forwarding strategies THMF (transmit maximum hop first) by applying the forwarding queue priority to reduce of delay transmisson and increase the delivery rate to achieve better performance. In default condition the lower priority message are being send first but the higher priority is queue, so delay transmission occurs in the network. The TMHF scheme utilizes network resources by giving priority to the message with higher of hop.

Ramanathan et. al. [6] evaluate the PRioritized EPidemic (PREP) routing protocol for delay tolerant network. This algorithm prioritizes bundles based on cost to destination, source and expiry time. PREP value is decrease with increasing distance from the destination. PREP has several advantages including keeps the storage and banwidth maximally utilized, dropping only when necessary. The comparison between epidemic routing and PREP routing show that PREP's delivery ratio is higher than epidemic routing.

Kiranmayi [7] propose optional control to overcome the problem in default epidemic routing. The default epidemic routing require more storage, more bandwith and nodes power due to sending the same messages many times. He overcome these problems with implement the epidemic with antipackets, epidemic with encounter count (EC) and epidemic with time to live (TTL). The epidemic with anti-packets was implemented to manages the buffer level when the buffer size is more. The EC deals with discarding of bundles when no more space for new bundles. The TTL deals with how much time the bundles can alive in the nodes buffer. The combination of EC and TTL can achieve more good performance.

\section{ORIGINALITY}

One of the mechanism of sending packet or bundle to carrier is based on Epidemic routing algorithm. Bundles are send to every carrier as copy. Then, one of them will forward the bundle to next DTN node or destination node. After bundle arrive at destination node, bundle list in carrier that forward the bundle will be 0 but not in other carrier. Basically bundle list of carriers will be 0 while the expiration time is up. So by using classic Epidemic algorithm to forward the bundle, will make network traffic increase because of the concept of Epidemic that send bundle to all DTN node as copy.

Although it flooding the network by sending a copy of bundle to all node, the nodes that receive bundle from source that actually for destination can not open the bundle. So another effect of Epidemic routing is make bundle's storage capacity on DTN node not efficient. To make Epidemic algorithm work more efficient we propose a new mechanism to forward the bundle to the destination using one choosen courier.

We call it Epidemic routing based signal level. First, source node will capture signal level of every DTN node that in coverage area of source node. We use iw command on Linux to capture signal level and compare it with 
another. The node with biggest signal level means that node is near to source node because in wireless communication, there are many factor that make signal level drop like obstacle or distance between node that communicate. The node with biggest signal level will be choosen as carrier that receive a copy bundle from source and forward it to next node or destination node. Every node will capture information about signal level of neighboor node. See Figure 3 about mechanism of sending bundle from source to choosen carrier. According to Epidemic routing based on signal level, bundles from source node take the path to the destination through DTN node 3 because it has the best signal level than another DTN node and forward it to next node or destination. By modifying classic Epidemic routing algorithm before injecting bundle to all node, we insert a script that compute the signal level then compare them to get the greatest one to receive bundle as carrier.

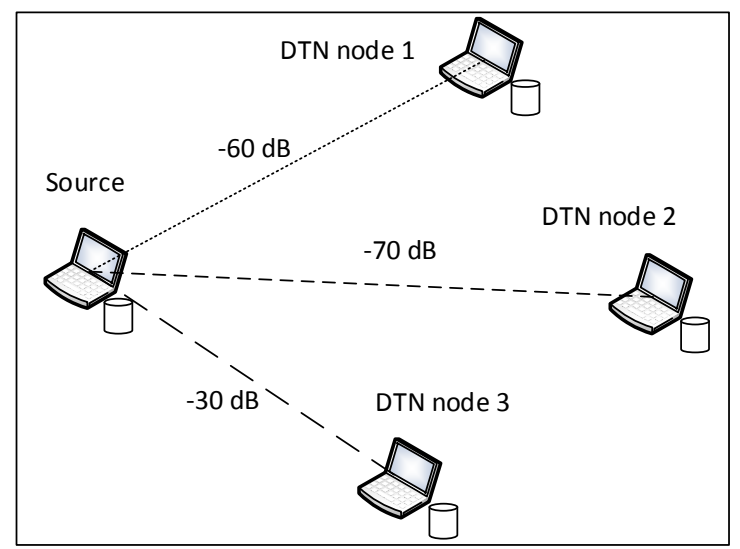

Figure 1. Epidemic routing based signal level.

DTN nodes in our test are mobile, so it is possible for DTN node 2 or DTN node 1 to receive a packet from source and act as carrier to forward the packet to next node or destination node as long as it has the highest level signal. In our test, we set the capture time every node to get information about level signal of their neighbor node every 1 second and it will be processed to recompute and take decision which node that will be chosen as carrier. So with this scenario can reduce bandwith usage.

\section{Epidemic Routing for Delay Tolerant Network \\ 4.1 Delay Tolerant Network (DTN)}

Delay tolerant network (DTN) is a protocol that aims to provide efficient communication between source node to destination node with no guaranted continuous connectivity. DTN is the area of networking which addresses challenges in disconnected, disrupted networks without end-toend connection. DTN is designed to operate effectively over extreme distances such as those encountered in space communications or on an interplanetary scale. Another characteristic of DTN network are : 
- Intermittent Connectivity : In this area, connectivity between source and destination is no guarantee still connected or available during communication. Sometimes source or destination is mobile and could make source and destination are out of range.

- Long or Variable Delay : Another effect of Intermittent Connectivity area is long propagation delay because mobility and obstacle.

- Limited Resources : The nodes in DTN is mobile devices with limited resources. Because with store and forward principle the current node have to safely store the bundle until the link to the destination is available.

The DTN do not have any topological information, uncertain between node. The DTN architecture use mobile carrier nodes for carrying and forwarding the messages and make communication possible among these nodes. The concept of DTN is store and forward message switching. If the link between source and next node available, bundles will be forwarded. But if link between node and destination unavailable, the bundles will be stored on that node. In other words Messages are buffered before they are forwarded to next node. Illustration of store and forward is shown in Figure 2[8]. The epidemic routing protocol explore all available communication paths with another neighbor nodes to transmit the messages.

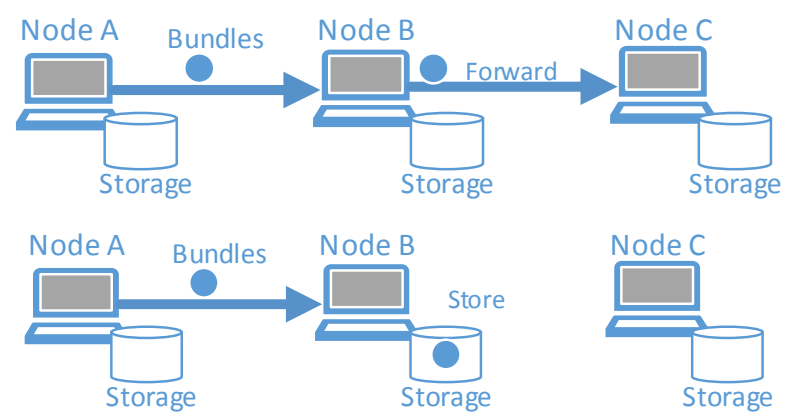

Figure 2. Store and Forward concept[8].

\subsection{Routing in Delay Tolerant Network}

Although DTN is addressed to be tolerant of delay, a routing protocol with better performance will maximizing packet delivery rate and minimizing the delivery latency. Routing is a process that data packets are transferred from source to the destination. Routing in DTN have several of issues, including buffer space, energy, reliability, processing power and security [9]. When the communication link is unavailable, current node must be buffered of the messages for long periods. So, the carrier mobile node device require enough buffer space to store the data packets. With good routing performance can decrease delay of pending messages which be stored in the carrier nodes. A Routing protocol in DTN can also decrease the energy to sending and receiving when the node is mobile. The objective of traditional routing has been to select the shortest path with minimum 
number of hops. Routing goal in DTN is to maximize probability of the successful delivery ratio of the packet data.

The routing protocols in DTN based on the type of knowledge used for routing can be classified into flooding based approaches and forwarding based approaches [10]. The flooding based routing approach, a node copies the message to all the nodes that connected with it. This is done to increase the probability of delivery of data to the destination. A epidemic routing protocol is a flooding based algorithm to maximize message delivery rate and minimize message latency. But, with epidemic routing can consume more network resources because for a single message to be delivered the whole network could be holding so many copies of that message. Whereas, a history based routing approach utilizes the history of encounters between nodes, to make a routing decision.

\subsection{Epidemic Routing}

Epidemic routing is a flooding routing protocol. It is easiest dynamic routing protocol based on replication scheme. This routing protocol is used if the network has absolutely no knowledge about the network. All of the devices act as the relay node (carrier node). Each device (source and carrier node) send the replicas packet to all contactable nodes. In other words the epidemic routing floods the message to all its neighbours. Then it relies on neighbors to transmit messages through flooding to increase delivery rate to the destination with maximal spreading of the messages throughput to the network. The epidemic routing scenario is illustrated in Figure 3[8].

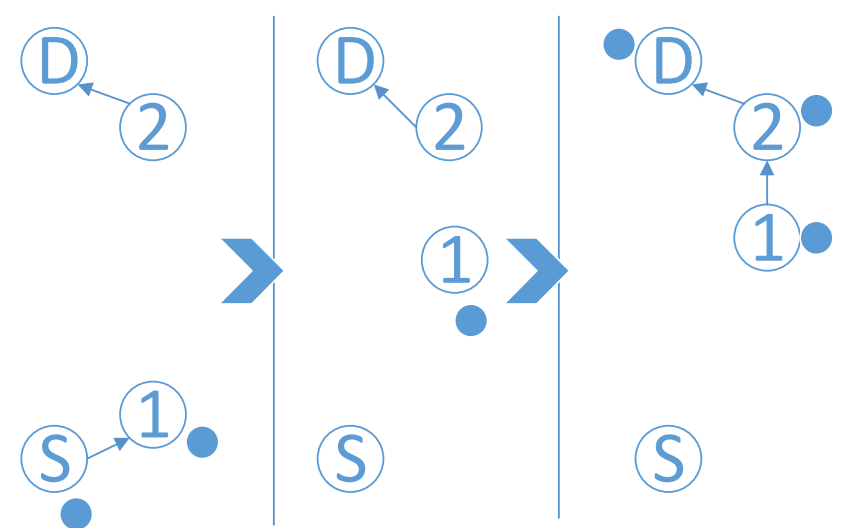

Figure 3. The epidemic routing scenario[8]

Packet from source to destination flooded through node 1 because only node 1 that covered by source node. Then node 1 move to node 2 , so packet from node 1 forwarded through node 2 and finally sent to destination node because node 2 and destination node are in coverage. The epidemic routing is able to achieve to high packet delivery rate by flooding the packets on the network. But this routing has low performance when each node has limited resources and limited bandwidth. 


\section{MEASUREMENT RESULT}

Our implementation using DTN2 framework which be developed by Delay Tolerant Networking Research Group (DTNRG). It works well in Ubuntu and Debian OS and support for mini PC like raspberry pi both 32 bit or 64 bit processor architecture. There are many application in DTN2 and we use one of them called dtncp that used on source node to send a file to destination node and dtnrecv as the pair that used on destination node to pick up the packet. We assume that source node and destination node are out of range. So we need a DTN nodes as carrier in the intermittent area to carry the packet from source node and deliver it to destination node. We use a DTN node more than one and set the time expiration of packet in long duration to make packet still available and arrive at destination. Figure 4 show the illustration of DTN network in our evaluation.

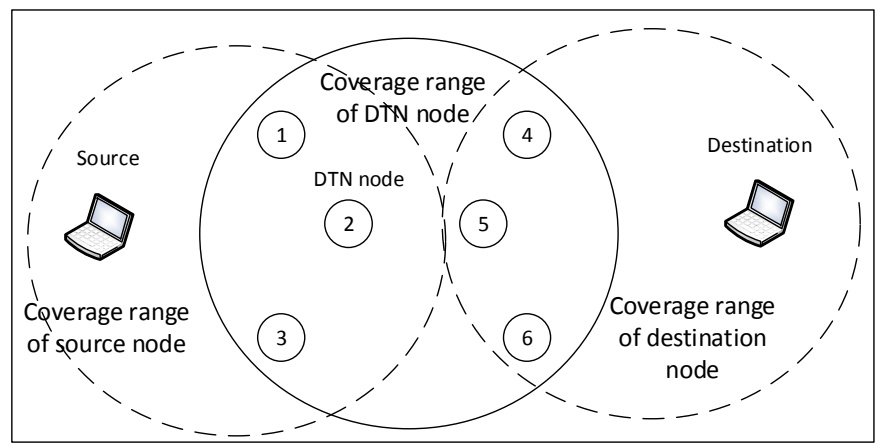

Figure 4. Illustration of DTN network.

There are three areas in our test. First area is source node that can reach DTN node 1,2 , and 3. Another DTN nodes and destination node are unreachable. So packet from source node should take path between DTN node 1,2 , or 3 . Second area is area that covered by DTN node 1,2 , and 3 . They are source node, DTN node 4,5 , and 6 . The connection between DTN node 1,2 , and 3 and destination is unreachable, so packet from DTN node 1 , 2 , or 3 should forwarded to DTN node 4,5 , or 6 . The third area is area that covered by destination node. They are DTN node 4,5 , and 6 . So, destination node can communicate with DTN node 4,5 , and 6 to receive the packet from one of them. Figure 3 show the parameter that used on measurement.

Table 1. Parameter Simulation

\begin{tabular}{|c|c|}
\hline Number of nodes & 8 \\
\hline Topology & 2-hop, 3-hop, 4-hop \\
\hline Wireless mode & Ad-hoc \\
\hline Size of packet & 20 bytes (ping) \\
\hline Routing algorithm & Epidemic based signal level \\
\hline Expiration packet & 12 second \\
\hline Parameter masurement & QoS(delay and throughput) \\
\hline
\end{tabular}


Our simulation used 2 nodes as source and destination and three nodes as carrier in 2-hop topology and four nodes as carrier in 3-hop topology. The specifications of nodes that used on measurement are show in Table 2.

Table 2. Spesification of nodes

\begin{tabular}{|c|c|c|}
\hline Node type & Source / Destination & DTN node \\
\hline Board type & Laptop & Raspberry Pi \\
\hline Processor & AMD A8 2.3 GHz & Quad Core Broadcom \\
\hline Memory & 8 GB DDR 3 & 1 GB DDR 3 \\
\hline Storage & $300 \mathrm{~GB}$ & 16 GB Micro SD \\
\hline OS & Ubuntu 14.04 x86 & Raspbian Wheezy \\
\hline Interface & WLAN 802.11b/g/n & WLAN $802.11 \mathrm{~b} / \mathrm{g} / \mathrm{n}$ \\
\hline
\end{tabular}

The result of dtnping when we use epidemic and priority epidemic routing protocol is show in Figure 5. Figure 6 show the comparation between Epidemic and priority epidemic routing in 2-hop topology.

Table 3. dtnping latency result.

\begin{tabular}{|c|c|c|c|c|}
\hline \multirow{2}{*}{ Sequence } & \multicolumn{2}{|c|}{ Classic Epidemic (ms) } & \multicolumn{2}{c|}{ Priority Epidemic (ms) } \\
\cline { 2 - 5 } & 2-hop & 3-hop & 2-hop & 3-hop \\
\hline 1 & 818.7 & 852 & 708.9 & 674.3 \\
\hline 2 & 773.7 & 801.5 & 611.9 & 623.4 \\
\hline 3 & 699.5 & 696.1 & 585.7 & 660.1 \\
\hline 4 & 717.3 & 701.8 & 598 & 739.1 \\
\hline 5 & 671.2 & 651.4 & 625.5 & 632.1 \\
\hline 6 & 616.2 & 607.3 & 600.9 & 708.4 \\
\hline 7 & 757.7 & 760.3 & 654.3 & 652.3 \\
\hline 8 & 789 & 880.1 & 615.2 & 735.4 \\
\hline 9 & 766.3 & 788.5 & 643.4 & 758.9 \\
\hline 10 & 735.7 & 767.9 & 729.9 & 786.3 \\
\hline Average & 734.53 & 750.69 & 637.37 & 697.03 \\
\hline
\end{tabular}

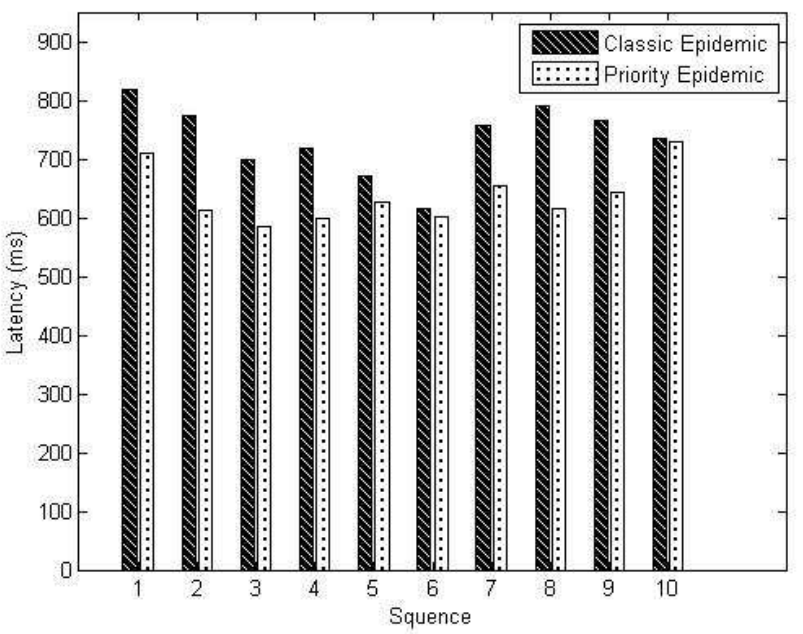

Figure 5. dtnping result 2-hop topology. 
From Figure 5, we could see that classic epidemic routing make latency higher than priority epidemic. The priority routing has lower latency in all sequence. The average different latency between them is $97.16 \mathrm{~ms}$. Figure 6 show the comparation between classic epidemic and priority epidemic in 3hop topology.

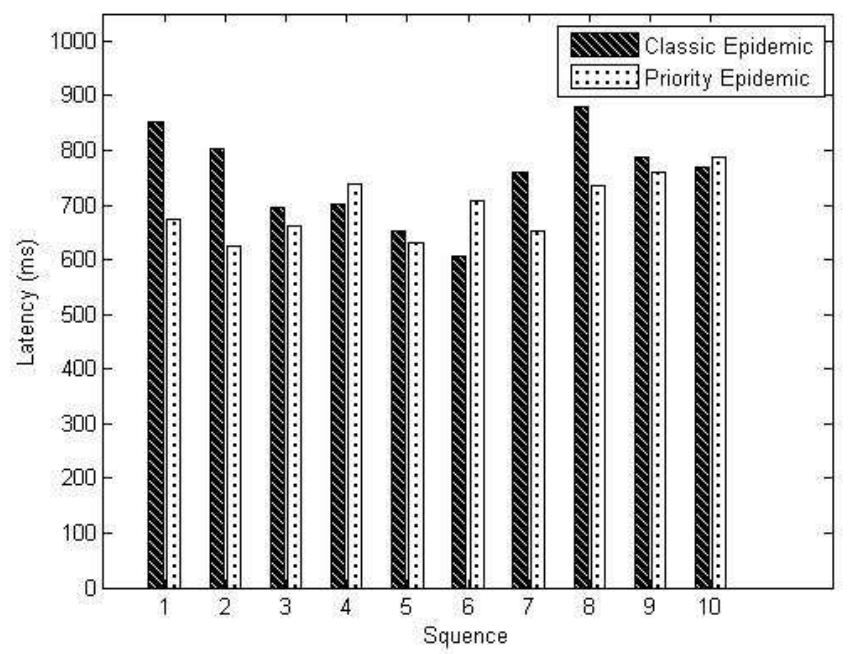

Figure 6. dtnping result 3-hop topology.

From figure 6, we could see that classic epidemic routing make latency higher than priority epidemic. The average different latency between them is $53.66 \mathrm{~ms}$. Table 4 show the throughput and delay transmission result between classic epidemic and priority epidemic. We also meassure the throughput with several size file. In this measurement use file with size 200 $\mathrm{KB}, 400 \mathrm{~KB}, 600 \mathrm{~KB}, 800 \mathrm{~KB}$ and $1 \mathrm{MB}$. The file is sent from server node with dtnperf-client command. The packet data is received in the destination node with dtnperf-server command. The principle of dtnperf command is devide the message became several bundles with size $50 \mathrm{~KB}$. In 2-hop topology with classic epidemic routing and priority epidemic routing, the delay transmission and throughput are like in Table 4.

Table 4. Delay transmission and throughput of classic epidemic routing and priority epidemic routing in 2-hop topology

\begin{tabular}{|c|c|c|c|c|}
\hline \multirow{2}{*}{$\begin{array}{c}\text { File } \\
\text { size }\end{array}$} & \multicolumn{2}{|c|}{ Classis Epidemic } & \multicolumn{2}{c|}{ Priority Epidemic } \\
\cline { 2 - 5 } & Delay Transmission & Throughput & Delay Transmission & Throughput \\
\hline $200 \mathrm{~KB}$ & $14.57 \mathrm{~ms}$ & $0.155 \mathrm{Mbps}$ & $3.78 \mathrm{~ms}$ & $0.427 \mathrm{Mbps}$ \\
\hline $400 \mathrm{~KB}$ & $25.71 \mathrm{~ms}$ & $0.135 \mathrm{Mbps}$ & $10.24 \mathrm{~ms}$ & $0.268 \mathrm{Mbps}$ \\
\hline $600 \mathrm{~KB}$ & $35.44 \mathrm{~ms}$ & $0.144 \mathrm{Mbps}$ & $13.72 \mathrm{~ms}$ & $0.355 \mathrm{Mbps}$ \\
\hline $800 \mathrm{~KB}$ & $42.70 \mathrm{~ms}$ & $0.209 \mathrm{Mbps}$ & $19.26 \mathrm{~ms}$ & $0.362 \mathrm{Mbps}$ \\
\hline $1 \mathrm{MB}$ & $29.94 \mathrm{~ms}$ & $0.375 \mathrm{Mbps}$ & $67.94 \mathrm{~ms}$ & $0.128 \mathrm{Mbps}$ \\
\hline
\end{tabular}

We could see that throughput when we use priority epidemic routing is higher than classic epidemic routing. The total throughput in one communication in classic epidemic routing is $0.155 \mathrm{Mbps}$ for send file with 
size $200 \mathrm{~KB}$, but in priority epidemic routing it is $0.427 \mathrm{Mbps}$ for same size file. The delay transmission to send files with size $200 \mathrm{~KB}$ require $14.57 \mathrm{~ms}$ for classic epidemic routing and 3.78 when uses priority epidemic routing. In 3-hop topology, the result of throughput when we use epidemic routing is like Table 5.

Table 5. Delay transmission and throughput of classic epidemic routing and priority epidemic routing in 3-hop topology

\begin{tabular}{|c|c|c|c|c|}
\hline \multirow{2}{*}{$\begin{array}{c}\text { File } \\
\text { size }\end{array}$} & \multicolumn{2}{|c|}{ Classis Epidemic } & \multicolumn{2}{c|}{ Priority Epidemic } \\
\cline { 2 - 5 } & Delay Transmission & Throughput & Delay Transmission & Throughput \\
\hline $200 \mathrm{~KB}$ & $12.18 \mathrm{~ms}$ & $0.16 \mathrm{Mbps}$ & $6.15 \mathrm{~ms}$ & $0.36 \mathrm{Mbps}$ \\
\hline $400 \mathrm{~KB}$ & $19.22 \mathrm{~ms}$ & $0.28 \mathrm{Mbps}$ & $10.35 \mathrm{~ms}$ & $0.37 \mathrm{Mbps}$ \\
\hline $600 \mathrm{~KB}$ & $18.94 \mathrm{~ms}$ & $0.27 \mathrm{Mbps}$ & $14.62 \mathrm{~ms}$ & $0.36 \mathrm{Mbps}$ \\
\hline $800 \mathrm{~KB}$ & $46.06 \mathrm{~ms}$ & $0.23 \mathrm{Mbps}$ & $21.66 \mathrm{~ms}$ & $0.32 \mathrm{Mbps}$ \\
\hline $1 \mathrm{MB}$ & $118.07 \mathrm{~ms}$ & $0.15 \mathrm{Mbps}$ & $27.49 \mathrm{~ms}$ & $0.30 \mathrm{Mbps}$ \\
\hline
\end{tabular}

We could see that throughput when we use priority epidemic routing in 3-hop topology is higher than classic epidemic routing. The total throughput in one communication in priority epidemic routing is $0.36 \mathrm{Mbps}$ for send file with size $200 \mathrm{~KB}$, but in classic epidemic it's only $0.16 \mathrm{Mbps}$. The delay transmission to send files with size $400 \mathrm{~KB}$ require $19.22 \mathrm{~ms}$ for classic epidemic routing and 10.35 when uses priority epidemic routing

\section{CONCLUSION}

Epidemic based on signal level routing could make traffic of network more eifficient than classic Epidemic routing because of filtering system in node before sending bundle to neighboor node. The advantage for DTN node is a storage capacity eifficiency because only one DTN node that receive bundle from source node. We try to break down the impact of signal level for another property like noise, transmission delay to get average of waiting time for bundle while deliver it from source to next node. The algorithm of sending bundle from source node to destination based on average waiting time and transmission delay based on research from Sushant Jain, Kevin Fall and Rabin Patra called Minimum Expected Delay (MED) routing[4]. MED was Dijkstra with time-invariant edge cost based on average edge waiting time. By using more complex of parameter to deliver bundle, hope will make DTN network more effective although DTN network give tollerant for delay.

\section{Acknowledgements}

The authors would like to thank Privacy Enhancement Technologies (PETs) Research Group members for valuable comments which helped to improve the exposition of our results. This research was supported in part by Penelitian Lokal PENS. 


\section{REFERENCES}

[1] Cerf, V., Burleigh, S., Hooke, A., Torgerson, L., Durst, R., Scott, K., Fall, K., and Weiss, H., Delay-Tolerant Network Architecture, RFC4838, 2007

[2] E. Husni, A. Wibowo, E-mail System for Delay Tolerant Network, International Conference on System Engineering and Technology, Bandung, Indonesia, September 11-12, 2012

[3] F. Zhang, J. Ma, D. Gao, X. Tan and Y. Liu, A Routing Protocol for DelayTolerant Network, International Conference on Engineering Technology and Application (ICETA),2015

[4] F. D. Rango, S. Amelio, P. Fazio, Epidemic Strategies in Delay Tolerant Networks from an Energetic Point of View: Main Issues and Permformance Evaluation, Journal of Networks, Vol. 10, No. 1, pp 4-14, Academy Publisher, January 2015

[5] N. Bajpai, Sandhya,Priority Scheme : Routing in TMHF for Epidemic Routing Protocol, International Journal of Scientific \& Engineering Research Volume 3, Issue 12, ISSN : 2229-5518, December 2012

[6] R. Ramanathan, R. Hansen, P. Basu, R. Rosales-Hain, R. Krishnan,Prioritized Epidemic Routing for Opportunistic Networks, Proceedings of the 1st international MobiSys workshop on Mobile opportunistic networking, ISBN: 978-1-59593-688-2, pp 62-66, New York, USA, 2007

[7] S. Wang, MHR. Khouzani, B. Krishnamachari, F. Bai,Optimal Control for Epidemic Routing of Two Files with Different Priorities in Delay Tolerant Networks, American Control Conference (ACC), Print ISBN : 978-1-4799-8685-9 , pp 1387 - 1392, Chicago, July 2015

[8] A. Zainudin, A. Sudarsono, K. P. Pambudi,Performance Analysis of Dynamic Routing for Delay Tolerant Network Architecture using DTN2 Framework,SNTT,UGM, Yogyakarta,2015

[9] N. Metha, M. Shah,Performance of Epidemic Routing Protocol in Delay Tolerant Network : A Comparative Survey,International Journal of Future Generation Communication and Networking, Vol. 7, No. 1, pp 151-158,2014

[10] S. Shukla, A. Munjal, Y. N. Singh,Routing protocol Approaches in Delay Tolerant Networks, International Conference on Educational and Information Technology MCNC, pp 91-95,2015 